Category: Simulation and Skills competency

\title{
Does virtual simulation improve confidence levels in final year medical students?
}

\author{
Nichols, C., Scully, A., Trendall, L., Ong, G.S., Goodson, M. \\ Newcastle University Medicine, Malaysia
}

\section{Background:}

Final-year students at Newcastle University Medicine Malaysia (NUMed) are currently unable to enter clinical teaching facilities due to the pandemic. Students would previously have shadowed junior doctors providing opportunities to gain confidence in undertaking the roles of a doctor. A virtual simulationbased training exercise was developed to simulate a junior doctor being on call. Evidence shows that simulation improves confidence in healthcare professionals (1). However, limited research exists into the value of online simulation. This study aims to evaluate medical students' confidence levels in areas of clinical practice before and after an online simulation.

\section{Method:}

35 randomly selected final-year NUMed medical students underwent online simulation training in January 2021. The simulation scenarios, previously used in face-to-face teaching for more than 300 students were converted for online use, mimicking a 'Bad day on call' for a junior doctor. Students were given a questionnaire to rate their confidence when dealing with three specific scenarios (breaking bad news, prioritising tasks and working under pressure) before and after completing a 140-minute simulation and a post-simulation debrief. Statistical comparisons of self-rated confidence before and after simulation training were undertaken using chi square analysis.

\section{Results:}

35 students participated in this pilot online study. A statistically significant difference in pre (mean $49.57+2.87$ ) and post (mean 63.00+2.99) simulation confidence scores for working under pressure $(p=0.028)$ was reported. A statistically significant difference $(p=0.001)$ between pre $(63.85+1.9)$ and post $(70.86+2.25)$ simulation confidence in prioritising tasks was also reported and mean scores were significantly different between male and female students $(p=0.01)$. No statistically significant differences in pre and post simulation confidence for breaking bad news were found.

\section{Conclusion:}

Virtual simulation can improve confidence levels about on-call shifts in final-year medical students. In situations where students cannot participate in face-to- face training, virtual simulation of on-call shifts may represent an acceptable replacement.

Keywords: Virtual Simulation, student's confidence

12 